\title{
Labyrinthe
}

3| 1999

Numéro 3

Sources et méthodes historiques

\section{Appropriation et pratiques de la lecture}

Les fondements méthodologiques et théoriques de l'approche de

l'histoire culturelle de Roger Chartier

\section{Dorothea Kraus}

\section{(2) OpenEdition}

Journals

Édition électronique

URL : http://journals.openedition.org/labyrinthe/56

DOI : $10.4000 /$ labyrinthe. 56

ISSN : 1950-6031

Éditeur

Hermann

Édition imprimée

Date de publication : 15 avril 1999

Pagination : 13-25

Référence électronique

Dorothea Kraus, «Appropriation et pratiques de la lecture », Labyrinthe [En ligne], 3| 1999, mis en ligne le 07 février 2005, consulté le 19 avril 2019. URL : http://journals.openedition.org/labyrinthe/56 ; DOI : $10.4000 /$ labyrinthe.56

Ce document a été généré automatiquement le 19 avril 2019.

Propriété intellectuelle 


\title{
Appropriation et pratiques de la lecture
}

\author{
Les fondements méthodologiques et théoriques de l'approche de
} l'histoire culturelle de Roger Chartier

\author{
Dorothea Kraus
}

1 «Le plus souvent, le seul indice de l'usage du livre est le livre lui-même. De là, les sévères limites imposées à toute histoire de la lecture. De là, aussi, son impérieuse séduction ${ }^{2}$. » C'est ainsi que Roger Chartier décrit les difficultés et les attraits de son champ de travail sur l'histoire culturelle, qui relie l'histoire du livre, la critique des textes et la sociologie de la culture. Lorsqu'il caractérise expressément son approche comme une " histoire culturelle du social ", il s'attaque au modèle traditionnel d'une « histoire sociale de la culture » qui part du principe que les clivages culturels sont avant tout l'expression des hiérarchisations sociales. À cette division dérivée des critères socioprofessionnels ou proprement politiques, Roger Chartier oppose une réévaluation différente des principes sociaux et culturels de différenciation : l'appartenance à un sexe, à une génération, les convictions religieuses, les traditions d'éducation et de formation en font par exemple partie $^{3}$. Dans le cadre de son champ de recherches qui porte sur une histoire du livre et de la lecture, il montre que la circulation de l'imprimé dépasse les frontières étroites des catégories sociales. Afin de saisir des partages sociaux plus fermes à l'intérieur d'une société, Roger Chartier propose une démarche qui prend comme point de départ la variété des pratiques de lecture effectuées par des " communautés de lecteurs ${ }^{4}$ » et les processus multiformes d'appropriation des textes.

2 Les livres n'ont pas, selon lui, un « sens » inhérent et absolu que le lecteur devrait retrouver. La construction de la signification ne s'effectue que par le lecteur : « [...] les réceptions sont toujours des appropriations qui transforment, reformulent, excèdent ce qu'elles reçoivent $^{5}$. " La construction du sens dépend des variations du texte, de sa forme imprimée et des différentes pratiques de la lecture, silencieuse ou à voix haute, en groupe ou individuelle, avec expérience ou hésitation. La " discipline » et " l'invention » caractérisent ce processus, au cours duquel la lecture peut s'affranchir des contrôles et des limitations que les dispositifs du texte lui imposent, car aucun texte n'est écrit 
indépendamment d'influences extérieures et d'intentions. Le chemin de la diffusion des textes est tout aussi complexe, car il ne mène pas de façon linéaire des hautes couches de la société aux couches inférieures, mais il se déroule selon une relation complexe d'imitation et d'adaptation'.

3 Les spécialistes de littérature se sont déjà beaucoup interrogés sur la difficulté à saisir de manière analytique la réception d'un texte. Le cas de «l'école de Constance ", très célèbre en Allemagne, autour de Wolfgang Iser et de Hans Robert Jauss, prend ici valeur de paradigme. Dans le caractère abstrait de ses appuis méthodologiques, « l'esthétique de la réception " demeure, ainsi que le souligne Roger Chartier, prisonnière d'une interprétation immanente de l'œuvre, et cela malgré sa focalisation sur le lecteur. Le défi de Roger Chartier est autre : saisir le lecteur historique réel dans son « horizon d'attente ${ }^{7}$ » ainsi que les possibilités d'une réception historique du texte.

4 Comment peut-on tenter cette entreprise rendue difficile par l'état des sources? Quelles étapes d'analyse et quelles modalités méthodologiques Roger Chartier propose-t-il afin d'y parvenir ? Comment relie-t-il le monde du texte et le monde du lecteur?

5 Ces questions se trouvent au cœur de l'analyse qui va suivre. Deux notions-clés doivent être d'abord étudiées : « appropriation » et " pratiques de la lecture ». Leurs définitions et leurs explicitations théoriques seront complétées par des exemples extraits d'études de cas. Roger Chartier lui-même développe ses apports méthodologiques et sa conceptualisation à partir de la pratique du travail historique.

6 Cette étude ne peut avoir pour ambition de rendre compte de la complexité de l'approche de Roger Chartier. Il s'agit bien plutôt de définir l'histoire de la lecture comme un élément constitutif d'une histoire culturelle du social et d'interroger les outils méthodologiques et d'analyse qu'il propose. Comme ses principes et ses concepts essentiels ont été développés et mis en œuvre dans les années 80 et n'ont connu par la suite que des précisions, des affinements et pour partie des élargissements, la chronologie de ses œuvres peut ici être laissée de côté. On se concentrera plutôt sur l'analyse et l'ordonnancement systématiques des éléments les plus importants de son champ de travail.

$7 \quad$ Le concept de Roger Chartier d'appropriation des textes est intimement lié à l'examen des pratiques de la lecture : «L'appropriation telle que nous l'entendons vise une histoire sociale des usages et des interprétations, rapportés à leurs déterminations fondamentales et inscrits dans les pratiques spécifiques qui les produisent ${ }^{8} . »$

8 Les " déterminations fondamentales » de l'appropriation comportent d'une part les différences dans les pratiques et les compétences de lecture elles-mêmes, et se rapportent d'autre part aussi aux modalités de la production et de la présentation du texte, au contexte social dans lequel les livres sont écrits, imprimés et lus. Lire est toujours pour Roger Chartier " une pratique incarnée dans des gestes, des espaces, des habitudes ${ }^{9}$ ». Un regard posé sur les thèses centrales de sa monographie sur Les Origines culturelles de la Révolution française devrait montrer de quelle manière l'appropriation dépend fondamentalement de ses déterminations culturelles et des pratiques de la lecture.

9 Le titre de son ouvrage ne doit pas induire en erreur : il ne cherche pas à savoir si la Révolution française a eu des origines davantage "culturelles " que par exemple économiques ou politiques. Il s'intéresse plutôt au rôle que la lecture d'un nombre toujours croissant de livres politiques et philosophiques a joué dans la Révolution, sur fond d'évolution d'ensemble de la société dans la longue durée. Roger Chartier s'attaque 
ici à la thèse défendue en 1933 par Daniel Mornet, selon lequel la Révolution serait une conséquence nécessaire et univoque de la diffusion et de l'intériorisation des idées des Lumières au XVIII ${ }^{e}$ siècle, qui furent réalisées avant tout grâce au livre ${ }^{10}$. Pour lui, instaurer cette relation causale, ce serait reprendre à son propre compte la tentative de légitimation ultérieure effectuée par des révolutionnaires qui voulaient créer une continuité dans le développement historique par la référence aux Lumières : " En affirmant que ce sont les Lumières qui ont produit la Révolution, l'interprétation classique n'inverse-t-elle pas l'ordre des raisons et ne faudrait-il pas plutôt considérer que c'est la Révolution qui a inventé les Lumières en voulant enraciner sa légitimité dans un corpus de textes et d'auteurs fondateurs, réconciliés par-delà leurs différences vives, unis dans la préparation de la rupture d'avec l'ancien monde ${ }^{11}$ ?»

10 Selon Roger Chartier, les livres, qu'ils soient de nature philosophique, politique ou littéraire, ne s'inscrivent pas dans les têtes des hommes. Pour que ces livres puissent vraiment être lus et compris avec intérêt, certains changements d'opinions et de représentations sont nécessaires dans l'horizon de réception des hommes. Les évolutions décisives du XVIII e siècle sont pour lui la constitution d'une opinion publique critique, une attitude qui change vis-à-vis de la monarchie, une tendance croissante à la déchristianisation et la formation d'une sociabilité intellectuelle dans les salons et les cafés. Une nouvelle manière de lire se développa, moins entravée et plus critique, d'autant plus que le livre pénétrait dans toutes les pratiques sociales quotidiennes et perdait sa fonction originelle d'autorité, une fonction quasiment sacrale. Les pratiques de la lecture ont donc aussi été transformées avec les bouleversements de l'ensemble de la société. C'est ainsi qu'à travers la diffusion et le succès des livres des Lumières fut révélé un doute sur le mode de vie traditionnel, mais il n'y trouva pas sa source ${ }^{12}$.

11 L'appropriation ne s'effectue pas dans un vide social. Avec l'apparition d'une opinion publique, les pratiques de la lecture et la façon de concevoir la lecture dans le domaine social ont changé. La mise en pratique quotidienne d'une nouvelle attitude critique, dans les cafés et les salons, peut expliquer l'intérêt pour de tels livres : "Pourquoi ne pas penser, en effet, que l'engouement pour les « livres philosophiques » n'a été possible que parce que, préalablement, avait opéré un désinvestissement symbolique et affectif qui les rendait acceptables, compréhensibles, attendus ? Loin d'être les producteurs d'une telle rupture, ils en seraient donc les produits ${ }^{13} . »$

12 Les modes d'appropriation et, partant, l'accès des lecteurs au texte dépendent aussi selon Roger Chartier pour une large part des capacités de lecture des différentes communautés de lecteurs. Dans son esprit, les différentes modalités de la construction du sens et les séparations culturelles à l'intérieur de la société se reflètent dans ces pratiques de la lecture, silencieuse ou à voix haute, avec assurance ou hésitation ${ }^{14}$. La lecture silencieuse est de plus en plus répandue à partir du milieu du XIV siècle, de sorte que la lecture est de moins en moins soumise aux déterminations d'une situation d'oralité ${ }^{15}$. Le lecteur est dans une certaine mesure plus libre dans ses possibilités d'interprétation. Néanmoins, la lecture à haute voix demeure une aide pour ceux dont la lecture n'est pas aussi assurée et habile.

13 Cela montre clairement combien les compétences de lecture sont intimement liées aux normes de lecture prédominantes à l'intérieur d'une communauté de lecteurs et aux attitudes d'attente vis-à-vis de la pratique de la lecture. Roger Chartier démontre ainsi que la lecture silencieuse conduisit à l'émergence d'une sphère de l'intimité dans les élites sociales des $\mathrm{XVI}^{\mathrm{e}}$-XVIII ${ }^{\mathrm{e}}$ siècles en France, sans pour autant que les formes de 
l'oralité disparaissent complètement. La lecture à voix haute demeure, effectuée par un serviteur, ce qui n'est pas perçu comme contradictoire avec ce qui est privé ; la lecture à haute voix persiste aussi dans le cercle des amis ou sous forme de rituel familial. De manière comparable, l'utilisation d'imprimés était multiforme dans les couches inférieures, même si la lecture à voix haute répondait souvent aussi à la nécessité supplémentaire de transmettre l'écrit à ceux qui étaient incapables de lire. Seuls quelques types très restreints de livres étaient lus : des textes religieux, des romans de chevalerie ${ }^{16}$ ...

Deux formes contradictoires de lecture sont liées aux différentes compétences de lecture : la lecture intensive et la lecture extensive. Dans le cas de la lecture intensive, le lecteur se trouve face à un nombre réduit de livres, qui sont transmis de génération en génération. Des formes singulières de la pratique de la lecture et du traitement des textes en résultent. Ils sont entendus de manière récurrente, lus à voix haute en famille ou à l'église ; la répétition constante de textes déjà connus permet de mémoriser ce qui est entendu ; ce que l'on a appris par cœur est continuellement récité, pour soi ou pour les autres. La lecture de quelques livres que la mémoire peut convoquer devient un point de repère familier et quotidien, elle détermine par son intensité la pensée et la parole des hommes. Cette forme de lecture est avant tout caractéristique de la lecture de la Bible dans l'Allemagne réformée et dans l'Amérique puritaine. En France, ce sont surtout les livres bon marché et largement répandus de la Bibliothèque bleue ${ }^{17}$ qui de manière similaire ont été intériorisés par l'écoute puis récités de mémoire.

15 De cette lecture intensive se distingue une forme extensive de lecture. Elle se répand avec le nombre croissant de livres, la lecture individuelle et silencieuse et la valeur moindre accordée aux textes religieux. Le rapport avec le livre se situe à un niveau superficiel, est moins intensif, ce qui enlève l'aspect vital propre à la lecture intériorisée ainsi que le statut singulier dont bénéficie le livre en tant qu'objet rare ${ }^{18}$.

16 Mais la lecture intensive et la lecture extensive ne sont pas deux formes de lecture qui s'excluent mutuellement. Toutes deux peuvent coexister à l'intérieur d'une société et servir ainsi d'indicateur des différences culturelles ${ }^{19}$.

17 Les compétences de lecture et les formes de la lecture sont un champ problématique pour la recherche historique dans la mesure où elles sont très difficiles à interpréter à partir de sources de toute façon disparates et où les résultats ne peuvent être généralisés qu'avec précaution. Roger Chartier se réfère alors à d'autres domaines de recherches pour mieux appréhender les pratiques de la lecture et l'appropriation.

18 Roger Chartier utilise le concept de « lecteur implicite ", forgé par Wolfgang Iser dans le cadre de l'esthétique de la réception. Contrairement à W. Iser, pour qui le terme " implicite » ne se réfère pas à un lecteur réel, mais à un lecteur caché dans la structure du texte lui-même ${ }^{20}$, le lecteur implicite est pour lui le lecteur réel, auquel pense l'auteur lors de la production d'un texte. Cette conception correspond au concept de "lecteur visé21 ", venu de l'esthétique de la réception. Elle se préoccupe aussi des attentes et des compétences d'un public déterminé, dans la mesure où l'auteur et l'éditeur peuvent façonner le contenu et la forme d'un texte en tenant compte d'un groupe déterminé de lecteurs.

19 Du point de vue de l'histoire culturelle, l'auteur est un personnage historique aussi important que le lecteur. Par conséquent, il est également au centre d'une investigation qui cherche à déterminer ses caractéristiques socioculturelles. La relation entre texte et 
auteur peut ainsi être examinée de trois points de vue différents, à commencer par celui du rapport qui existe entre l'auteur dans son environnement social et l'arrière-plan de la genèse du texte. L'auteur se trouve aussi peu que le lecteur hors de son contexte culturel. Georges Dandin fut ainsi un travail de commande écrit par Molière à la demande de Louis XIV, ce qui éclaire aussi bien la dépendance de l'auteur vis-à-vis de la protection de la cour, que les effets de signification qui se nichent dans le texte lui-même ${ }^{22}$.

20 En deuxième lieu, la recherche doit se tourner vers les stratégies implicites ou explicites, les interprétations que l'auteur cherche à fournir. Ces dispositifs peuvent se trouver dans le texte lui-même sous la forme de structures poétiques ou narratives, de références à des conventions sociales ou littéraires qui orientent la compréhension dans une direction déterminée ${ }^{23}$. Mais ils peuvent aussi éclairer l'intention de l'auteur directement sous la forme d'avis au lecteur. Dans le Nouveau Secrétaire Français de François Colletets (une collection de modèles de lettres et d'indications pour la rédaction de lettres datée du XVII ${ }^{e}$ siècle), l'auteur s'adresse pour commencer aux lecteurs et met en valeur l'objectif de son livre : servir de modèle et de réservoir d'idées ${ }^{24}$.

21 Il faut cependant voir que même une formulation explicite de l'intention de l'auteur ne peut contraindre de manière impérieuse le lecteur à une interprétation déterminée. Malgré toutes les limitations et les restrictions, la lecture demeure toujours un acte créateur et rebelle. Pour caractériser la réception d'un texte, il faut aussi prendre en considération sa matérialité.

22 C'est le troisième axe de la relation entre auteur et texte : l'auteur détermine aussi, conjointement avec l'éditeur, les formes du texte, la matérialité du signe qui est aux yeux de Chartier d'une importance fondamentale pour la réception d'un texte.

23 «Identifier les effets de sens produits par ces formes matérielles est une nécessité pour qui veut comprendre, dans leur historicité, les usages et les interprétations dont un texte a été l'objet ${ }^{25} . »$

24 Si l'on suit la série des publications de Roger Chartier depuis les années 80, il semble alors que la forme matérielle du texte joue un rôle essentiel dans l'appréhension des modes d'appropriation et des pratiques de la lecture. Pour tous les problèmes de sources qui sont entraînés par le travail sur les lecteurs historiques, de nombreuses directions de recherches convergent vers une critique textuelle fondée sur l'analyse des formes matérielles : les compétences de lecture d'un public, la question de l'oralité, les possibilités de la construction du sens et enfin la recherche sur une histoire culturelle du social. Roger Chartier suit ainsi un chemin que l'esthétique de la réception n'a jamais emprunté, puisqu'elle prenait uniquement en considération le texte comme objet littéraire ou esthétique.

25 Dans la mesure où l'auteur conçoit son œuvre pour un « lecteur implicite », l'imprimeur ou l'éditeur détermine par la forme du texte une « lecture implicite ». Cette dernière sera d'autant plus significative pour la réception que la rédaction du texte et son impression seront séparées dans le temps. L'horizon d'attente du public retravaillé par les formes peut être diamétralement opposé à celui qui était originellement implicite et s'opposer ainsi - dans le sens donné par Roger Chartier à ce terme - au lecteur viséé ${ }^{26}$. Le concept de « forme » se rapporte dans cette perspective à différentes dimensions d'un ouvrage imprimé. Il s'agit d'une part de la forme extérieure du livre lui-même, au sens large comme au sens restreint. Le passage du volumen au codex et depuis les dernières décennies à l'écran d'ordinateur ${ }^{27}$ influe sur la construction du sens par le lecteur tout 
autant que le format d'un livre et la manière d'ordonnancer le texte, qu'il s'agisse d'un livre solidement relié, d'une collection peu coûteuse de feuilles reliées entre elles ou bien d'une feuille volante. L'agencement du texte peut aussi être un dispositif pour sa forme ${ }^{28}$. Lorsque les éditeurs de Troyes décidèrent, à partir d'œuvres originellement destinées à un public d'élite de lecteurs cultivés, de produire des éditions bon marché pour une vaste clientèle de lecteurs qui vivaient à la campagne et étaient moins cultivés, ils tinrent pour nécessaires de telles interventions eu égard à l'horizon d'attente des lecteurs. De nombreux textes de ces éditions devenues célèbres sous le nom de Bibliothèque bleue furent retravaillés de diverses manières : le nombre des chapitres fut accru pour créer des unités de lecture plus petites, même si la cohérence du contenu d'un texte devait en souffrir. Le texte lui-même fut raccourci et simplifié afin de mettre de côté des parties considérées comme superflues pour le public visé - c'est-à-dire ce qui n'était pas de manière stricte en relation avec le déroulement de l'action. Le choix des mots et la syntaxe furent aussi adaptés à l'horizon d'attente et aux compétences de lecture présumés. Le caractère unitaire du corpus de la Bibliothèque bleue reposait ainsi d'une part sur le choix de textes déterminés (vies de saints, contes, romans de chevalerie), et d'autre part sur des similitudes structurelles, qui mettent en lumière les horizons d'attente et les capacités des lecteurs supposés par les éditeurs ${ }^{29}$.

26 Les textes de la Bibliothèque bleue ne sont pas les seuls (beaucoup d'autres imprimés aussi) à laisser transparaitre dans leur forme et leur structure des signes qui indiquent si le texte était destiné à une lecture à voix haute ou bien à une lecture intensive. De courts chapitres, des paragraphes indépendants les uns des autres, des répétitions, des lignes d'action simples soutiennent l'exposé oralisation ${ }^{30}$. Dans le domaine de la forme du texte, il est aussi utile d'analyser la ponctuation elle-même, qui du reste peut fournir une indication sur une situation de récitation ou de lecture à haute voix - selon qu'elle corresponde plutôt à des pauses dans la parole et à l'intonation ou bien à la syntaxe. Il faut ajouter ici que les différences les plus infimes ou bien les fautes de ponctuation peuvent changer à elles seules le sens d'une phrase et ainsi son rôle dans le texte et la réception, comme Roger Chartier le montre avec l'exemple du Songe d'une nuit d'été de Shakespeare ${ }^{31}$.

27 L'exemple déjà évoqué des "Secrétaires » doit, pour conclure, permettre de voir dans quelle mesure la forme peut finalement avoir une influence massive sur le sens d'un texte pour le lecteur. Le Secrétaire de la Cour de Jean Puget de la Serre fut conçu comme une collection de modèles et d'indications de lettres pour un public cultivé, comme une aide pour correspondre avec des personnalités de rang plus ou moins élevé dans le respect de l'étiquette de la cour. Lorsque cette œuvre fut reprise dans la Bibliothèque bleue, elle atteignit un public qui n'était pas directement en relation avec la cour et avec ses usages. Comme dans le même temps les indications pour écrire furent ôtées du texte et que ne restèrent que les modèles de lettres, l'ouvrage ne put quasiment plus avoir d'usage pratique pour le public visé. L'attrait de ces textes résidait plutôt dans la curiosité pour l'inconnu, l'étranger ${ }^{32}$.

28 L'auteur, le texte, le livre et les lecteurs sont les quatre pôles entre lesquels se déploie le champ de travail de Roger Chartier sur une histoire du livre et de la lecture et qu'il cherche à relier dans le cadre d'une histoire culturelle du social. Le concept d'« appropriation " rend dans cette perspective non seulement possible de faire converger ces objets de recherche, mais aussi de les mettre en relation avec les pratiques de la lecture qui déterminent chaque appropriation et qui dépendent à leur tour des 
compétences de lecture d'une communauté de lecteurs, des stratégies de l'auteur, de la forme du texte. Quatre aspects sont essentiels pour chaque forme de réception : premièrement, la compréhension des déterminations par lesquelles la lecture pleine d'inventions des lecteurs est guidée et limitée ; deuxièmement, les effets de signification prédisposés par les textes (et donc par l'auteur) eux-mêmes ; troisièmement, les restrictions et les influences subies par le lecteur à travers les formes de la transmission du texte ; quatrièmement, les capacités d'une communauté de lecteurs ou bien les conventions de la lecture individuelle ${ }^{33}$.

29 L'appropriation et les pratiques de la lecture peuvent être utilisées comme des indicateurs des différences culturelles et sociales dans une société. La plus grande importance revient alors à la matérialité du signe : elle révèle tout autant les stratégies de l'auteur et partant la construction d'un lecteur implicite ou visé que les pratiques de la lecture qui s'inscrivent en elle. La délimitation par rapport à l'esthétique de la réception, que Roger Chartier accomplit ainsi, n'est pas sans poser de problèmes : sous des points de vue historico-critiques, une exagération des aspects formels d'un texte peut également conduire dans une impasse phénoménologique réductionniste, lorsque l'état des sources ne permet pas d'analyse plus globale. Roger Chartier reconnaît lui aussi le manque courant de sources explicites pour l'élucidation de l'appropriation et des pratiques de la lecture hors du texte lui-même ${ }^{34}$.

30 Un mode d'approche ordonné est par conséquent nécessaire pour appréhender le « monde comme représentation « à partir des pratiques culturelles de la lecture et de l'écriture. Roger Chartier part de certaines hypothèses : tout d'abord la prétention à une histoire totale doit être abandonnée pour reconnaître que l'appréhension des lecteurs et des lectures à l'intérieur d'une période historique donnée ne peut dans la plupart des cas être effectuée que sous la forme d'instantanés, d'études de cas, même si son approche et sa méthode peuvent revendiquer une validité générale. En second lieu, l'histoire de la lecture demeure soumise au problème de l'existence de sources adéquates, puisque des traces immédiates de lecteurs et de pratiques de la lecture ne sont que rarement décelables. Cela conduit en troisième lieu à un nécessaire examen critique du matériel présent afin d'échapper à la simplification historique. Effectuée avec la prudence qui s'impose, l'analyse des sources, mais aussi des études historiques déjà existantes sur la base de problématiques spécifiques, permet de caractériser l'historien Roger Chartier : les connaissances historiques apparemment les plus objectives et les plus vérifiables - parce qu'elles reposent sur une exploitation quantitative des sources - sont dépossédées de leur univocité par un regard critique qui met en lumière des différences. Roger Chartier ne cherche pas à développer de nouveaux modèles de compréhension englobants, mais à exploiter un matériel connu d'après des problématiques et des points de vue radicalement nouveaux, une tentative qui ne peut qu'enrichir l'histoire de la culture, quelle que soit par ailleurs l'orientation qu'on lui donne. 


\section{BIBLIOGRAPHIE}

\section{Roger Chartier}

CHARTIER Roger, « Alltägliches Schreiben und Lesen. Modelle und Praktiken des Briefschreibens im Frankreich des Ancien Régime », Leipziger Jahrbuch zur Buchgeschichte n², 1992, p. 11-34.

- Culture écrite et société. L'ordre des livres (XIV -XVIII ${ }^{\mathrm{e}}$ siècle), Paris, Albin Michel, 1996 (notamment «Communautés de lecteurs », p. 133-154, « De la fête de cour au public citadin », p. 155-204).

— «Die geschriebene Botschaft und ihre Rezeption. Vom Codex zum Bildschirm », Neue Rundschau , n 3, 1995, p. 117-131.

- «Die Praktiken des Schreibens », Geschichte des privaten Lebens, Bd. 3 : Von der Renaissance zur Aufklärung, éd. par Philippe Ariès et Roger Chartier, Francfort/Main, 1991, p. 115-166.

- « Du livre au lire », in R. Chartier, Pratiques de la lecture, Marseille, Rivages, 1985, p. 62-88.

— « Histoire et littérature »,Au bord de la falaise. L'histoire entre certitudes et inquiétude, Paris, Albin Michel, 1998, p. 269-288.

— « Le monde comme représentation », A.E.S.C., n 6, 1989, p. 15051520.

— Les Origines culturelles de la Révolution française, Paris, Seuil, 1990.

Esthétique de la réception

ISER Wolfgang, Der Akt des Lesens. Theorie ästhetischer Wirkung, Munich, 1994.

- Der implizite Leser. Kommunikationsformen des Romans von Bunyan bis Beckett, Munich, 1972.

\section{ANNEXES}

Traduit de l'allemand par Stéphane Péquignot pour Labyrinthe

\section{NOTES}

1.Cet article est extrait d'un mémoire rédigé en 1998 dans le cadre d'un séminaire sur " les méthodes de l'histoire sociale ", à l'université de Bielefeld. Une partie importante du texte original, qui étudiait le rapport entre l'approche chartierienne et celles que développent les spécialistes de littérature, est ici passée sous silence.

2.Roger Chartier, « Du livre au lire », Pratiques de la lecture, Marseille, Rivages, 1985, p. 87.

3.Roger Chartier, «Le monde comme représentation », in Annales E.S.C. n 6, 1989, p. 1511.

4.Id., «Communautés de lecteurs », Culture écrite et société. L'ordre des livres (XIV"e-XVIII

siècle), Paris, Albin Michel, 1996, p. 133-154.

5.Id., Les Origines culturelles de la Révolution française, Paris, Seuil, 1990, p. 30.

6.Id., Lectures et lecteurs d'Ancien Régime, Paris, Seuil, 1987, p. 16-17. 
7.Cf. par exemple Roger Chartier, « Alltägliches Schreiben und Lesen. Modelle und Praktiken des Briefschreibens im Frankreich des Ancien Régime », Leipziger Jahrbuch zur Buchgeschichte $\mathrm{n}^{\circ} 2,1992$, notamment p. 24 et 27.

8.Id., « Le monde comme représentation », loc. cit., p. 1511.

9.Ibid., p. 1510.

10.Daniel Mornet, Les Origines intellectuelles de la Révolution française 1715-1787 (1933), Paris, 1967, cité d'après Roger Chartier, Les Origines culturelles de la Révolution française, op. cit.

11.Roger Chartier, Les Origines culturelles de la Révolution française, op. cit., p. 14.

12.Cf. ibid., le chapitre « Les livres font-ils les révolutions?», p. 86-114.

13.Ibid., p. 109.

14.Roger Chartier, « Du livre au lire », loc. cit., p. 67.

15.Ibid., p. 67 et suiv.

16.Id., « Die Praktiken des Schreibens ", in Geschichte des privaten Lebens, Bd. 3 : Von der Renaissance zur Aufklärung, éd. par Philippe Ariès et Roger Chartier, Francfort/Main 1991, notamment les p. 144 et suiv. (en français, Histoire de la vie privée, tome 3 : De la Révolution aux Lumières, Paris, Seuil, 1986). Chartier y considère les représentations contemporaines de la lecture en commun tous les soirs dans les régions de campagne comme un «mythe ", comme l'expression d'une nostalgie répandue parmi les lecteurs urbains, car si les soirées étaient bien passées en société à la campagne, elles n'étaient que rarement l'occasion de lectures en commun, cf. Roger Chartier, « Du livre au lire », loc. cit., p. 77 et suiv.

17.Appelés ainsi d'après la reliure le plus souvent bleue des textes. Les livres bleus, des éditions bon marché des formes les plus variées de littérature, ont d'abord été imprimés à Troyes au XVII ${ }^{\mathrm{e}}$ siècle.

18. Roger Chartier, « Du livre au lire », loc. cit., p. 69-70.

19.Ibid., p. 72-73.

20.Wolfgang Iser, Der implizite Leser. Kommunikationsformen des Romans von Bunyan bis Beckett, Munich, 1972.

21.Id., Der Akt des Lesens. Theorie ästhetischer Wirkung, Munich, 1994, p. 58 et suiv.

22.Roger Chartier, « De la fête de cour au public citadin », Culture écrite et société. L'ordre des livres (XIV ${ }^{e}$-XVIII ${ }^{e}$ siècle), op. cit., p. 155-204.

23.Id., « Du livre au lire », loc. cit., p. 79.

24.Id., « Alltägliches Schreiben und Lesen », loc. cit., p. 23.

25.Id., Culture écrite et société, op. cit., p. 10.

26.Id., « Du livre au lire », loc. cit., p. 81-82.

27.Id., " Die geschriebene Botschaft und ihre Rezeption. Vom Codex zum Bildschirm », Neue Rundschau $\mathrm{n}^{\circ} 3$, 1995, p. 117-131. Sur la signification pour le lecteur du support du signe en voie de transformation, voir notamment p. 122 et suiv.

28.Id., « Du livre au lire », loc. cit., particulièrement p. 79-81.

29.Ibid., p. 82 et suiv.

30.Roger Chartier, « Histoire et littérature », Au bord de la falaise. L'histoire entre certitudes et inquiétude, Paris, Albin Michel, 1998, p. 269-288, notamment p. 274 et suiv.

31.Ibid., p. 284. Sur le problème de la ponctuation, voir de manière générale ibid., p.

277-278.

32.Id., « Alltägliches Schreiben und Lesen », loc. cit., p. 27.

33.Id., « Le monde comme représentation », loc.cit., p. 1510.

34.Cf. supra., note 2 . 


\section{RÉSUMÉS}

Ce travail envisage une analyse systématique de l'approche d'une histoire culturelle du social, telle qu'elle est effectuée par Roger Chartier, Professeur à l'E.H.E.S.S., Paris. En premier lieu, l'article vise à saisir le mode d'approche de Roger Chartier à l'intérieur de son champ de travail (l'histoire de la lecture) comme une méthode spécifique mais généralisable. Il s'agit d'abord de dégager ses catégories analytiques et ses modèles explicatifs, puis de les relier à l'histoire culturelle de l'Ancien Régime (XVI ${ }^{\mathrm{e}}-\mathrm{XVIII}^{\mathrm{e}}$ siècles). Des concepts centraux comme «l'appropriation » et les "pratiques de la lecture» doivent alors être examinés sous le double point de vue de leur réflexion théorique et de leur utilisation pratique par Roger Chartier ${ }^{1}$.

Appropriation and practices of reading. The methodological and theoretical fundaments of Roger Chartier's cultural history approach. - This study develops a systematic analysis of the approach to a cultural history of society, as it is done by Mr Roger Chartier, a professor at the School for Superior Studies in Social Sciences (E.H.E.S.S.) in Paris. In the first place, the article aims at grasping the approach of R. Chartier within his working field (i.e. the history of reading) as a method which is specific but which can also be generalised. It is first a matter of isolating the analytical categories and the explicative models of R. Chartier and then to connect them to the cultural history of the Ancient Regime (sixteenth-eighteenth centuries). Central concepts such as the " appropriation " and the " reading practices " have then to be examined from the double point of view of the theoretical reflection and their practical use by R. Chartier.

\section{AUTEUR}

\section{DOROTHEA KRAUS}

Dorothea KRAUS, née en 1974, étudie la philologie allemande, l'histoire et la philosophie à l'université de Bielefeld, en Allemagne. Elle prépare actuellement un mémoire de maîtrise interdisciplinaire sur la réception des œuvres littéraires allemandes, en relation avec la méthode historique de Roger Chartier. 